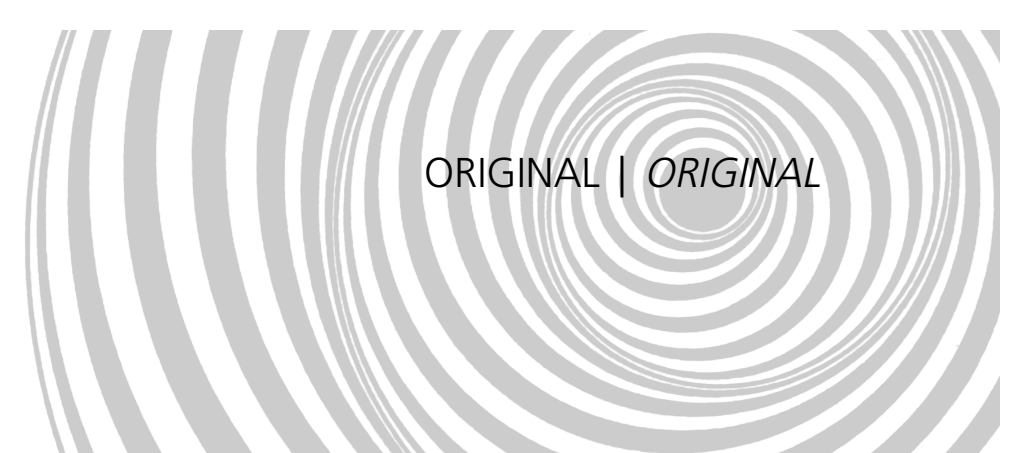

\title{
Perfil nutricional de recém-nascidos de mães adolescentes suplementadas com ferro, em diferentes concentrações, zinco e ácido fólico
}

\author{
Nutritional profile of newborns of adolescent mothers \\ supplemented with iron, in different \\ concentrations, zinc and pholic acid
}

Nadir do Nascimento NOGUEIRA' ${ }^{1}$

Antônio da Silva MACÊDO²

Joaquim Vaz PARENTE 2

Sílvia Maria Franciscato COZZOLINO³

RESUMO

O objetivo deste estudo foi avaliar o estado nutricional de recém-nascidos de mães adolescentes com até 18 anos de idade, de baixa renda, suplementadas durante a gravidez com ferro, em diferentes concentrações, zinco e ácido fólico. Estas mães fizeram pré-natal no Instituto de Perinatologia Social do Piauí, Maternidade Dona Evangelina Rosa, no município de Teresina, Piauí, Nordeste do Brasil. As adolescentes foram aleatoriamente distribuídas em cinco grupos: grupos I e II receberam quantidades constantes de ácido fólico $(250 \mu \mathrm{g})$ e diferentes concentrações de ferro, na forma de sulfato ferroso (120 e $80 \mathrm{mg}$, respectivamente); grupos III e IV receberam quantidades constantes de ácido fólico $(250 \mu \mathrm{g})$ associado ao sulfato de zinco $(5 \mathrm{mg})$ e ferro nas concentrações de 120 e 80 mg, respectivamente; e grupo V, considerado controle, recebeu apenas ferro (120 mg) (rotina da Instituição). A avaliação do estado nutricional de 74 recém-nascidos foi feita através de dados referentes à idade, peso, comprimento e perímetro cefálico, por ocasião do nascimento. De acordo com os resultados, as médias de peso $(3000 \pm 418 \mathrm{~g})$, comprimento $(48,07 \pm 2,15 \mathrm{~cm})$ e perímetro cefálico $(33,53 \pm 1,50 \mathrm{~cm})$ encontradas para os filhos de mães do grupo $\vee$ (rotina local) não diferem dos grupos de intervenção. Conclui-se que os recém-nascidos de adolescentes suplementadas com ferro em diferentes concentrações (120 e $80 \mathrm{mg}$ ), zinco e ácido fólico apresentaram um bom perfil nutricional. No entanto, não se observou, entre os grupos de intervenção e o controle, diferenças significativas na duração da gravidez ou no estado nutricional dos bebês.

Termos de indexação: recém-nascido, adolescência, gravidez, suplementação alimentar.

\footnotetext{
1 Departamento de Nutrição, Universidade Federal do Piaú. Campus Universitário Petrônio Portella, Bloco 13. Bairro Ininga, 64049-550, Teresina, PI, Brasil. Correspondência para/Correspondence to: N.N. NOGUEIRA. E-mail: nadirn@uol.com.br

2 Departamento Materno-Infantil, Universidade Federal do Piauí.

3 Departamento de Alimentos e Nutrição Experimental, Faculdade de Ciências Farmacêuticas, Universidade de São Paulo.
} 
194 | N.N. NOGUEIRA et al.

\section{A B S T R A C T}

The objective of this study was to evaluate the nutritional status of newborn infants of low income adolescent mothers, aged up to 18 years old, supplemented during pregnancy with iron, in different concentrations, zinc and folic acid. These mothers received prenatal care supervision in the Institute of Social Perinatology of Piauí, Dona Evangelina Rosa Maternity, located in the city of Teresina, Piauí, Northeast of Brazil. The adolescents were distributed at random into five groups: groups I and II received constant quantities of folicc acid (250 $\mu \mathrm{g}$ ) and different iron concentrations, in the form of iron sulfate (120 and $80 \mathrm{mg}$, respectively); groups III and IV received constant quantities of folic acid $(250 \mu \mathrm{g})$ associated to zinc sulfate $(5 \mu \mathrm{g})$ and iron in the concentrations of 120 and $80 \mathrm{mg}$, respectively; and group V, considered control, received only $120 \mathrm{mg}$ of iron (Institution routine). The evaluation of the nutritional status of 74 newborn infants was done through data referring to their age, weight, height and cephalic perimeter, on the occasion of their birth. According to the results, the averages of weight $(3000 \pm 418 \mathrm{~g})$, height $(48.07 \pm 2,15 \mathrm{~cm})$ and cephalic perimeter $(33.53 \pm 1,50 \mathrm{~cm})$ found in children of group $V$ mothers (local routine) did not present significant differences in relation to the intervention groups. The newborn infants of adolescents supplemented with iron in different concentrations (120 and $80 \mathrm{mg}$ ), zinc and folic acid, presented a good nutritional profile. However, it was not observed, between the intervention groups and the control, significant differences in the pregnancy duration or in the nutritional status of the babies.

Index terms: newborn infant, adolescence, pregnancy, supplementary feeding.

\section{NTRODUÇÃO}

Ferro e folato são dois nutrientes essenciais, mas estão freqüentemente deficientes em dietas de grupos populacionais. Ambos são necessários para o desenvolvimento normal do sistema hematopoiético, bem como para numerosos processos metabólicos. Desta forma, a deficiência de cada um ou de sua combinação poderá resultar em anemia, com conseqüências fisiopatológicas associadas (Bailey \& Cerda, 1988).

A deficiência de ferro vem sendo amplamente investigada, e sua elevada prevalência mundialmente considerada como decorrente de ingestão insuficiente para suprir as necessidades relativamente altas deste mineral durante a infância e a idade reprodutiva (Yip, 1995).

No que diz respeito à gravidez, a carência de ferro tem sido associada ao aumento da morbidade e da mortalidade de gestantes e do feto. A relação entre a anemia em mulheres grávidas e o risco de nascimento de crianças prematuras parece, estar especificamente vinculada a esta deficiência de (Scholl et al., 1992).

Com relação ao ácido fólico, segundo a Organización Mundial... (1987), uma dieta normal não é capaz de cobrir as demandas maiores das gestantes, sendo necessário o uso de suplementos de folato ou de alimentos enriquecidos. Se não houver a suplementação com esta vitamina, a prevalência de anemia megaloblástica, no final da gestação, pode oscilar entre $24 \%$ e $60 \%$. No caso específico das adolescentes grávidas, a depleção nutricional torna-se ainda mais séria, pois este grupo apresenta estoques nutricionais mais baixos, em virtude da demanda para o seu próprio crescimento e da tendência à baixa qualidade da dieta (Hediger et al., 1992).

A deficiência de folato na mulher grávida tem sido associada a uma série de complicações obstétricas, além da anemia megalobástica, tais como sangramento no terceiro trimestre, aborto, 
descolamento de placenta, prematuridade, baixo peso ao nascer, doença hipertensiva específica da gravidez, malformação fetal e defeitos no tubo neural (Rothman, 1970; Bailey \& Cerda, 1988; Daly et al., 1995).

Estudos sobre o papel metabólico do ferro e do folato durante a gravidez continuam sendo realizados; no entanto, os resultados ainda são controversos (Preziosi et al., 1997; Blot et al., 1999).

Com referência ao zinco, há evidências de que sua deficiência poderia ser um agente teratogênico em humanos e foi observada a ocorrência com elevada freqüência de defeitos no tubo neural, em regiões do mundo onde a carência desse mineral era prevalente (Sever \& Emanuel, 1973).

De acordo com pesquisas em cobaias; a deficiência de zinco em ratas grávidas tem resultado em uma variedade de malformações congênitas, bem como tem sido associada ao retardo no crescimento intra-uterino, diminuição no peso do cérebro fetal e anormalidades no comportamento (Sandstead, 1981, Apgar, 1992).

Além da associação entre estado nutricional materno e complicações na gravidez, uma outra vertente desta análise está ligada ao fato de a adolescente estar ou não em crescimento, por ocasião da concepção. Dados encontrados mostram que bebês de jovens em desenvolvimento têm menos peso, em compara-ção com os daquelas plenamente desenvolvidas e das mulheres mais velhas. No entanto, a hipótese da competitividade pelos nutrientes entre a grávida em crescimento e o seu feto é ainda bastante discutida (Scholl \& Hediger, 1993).

Neste contexto, cabe ainda apontar a influência da idade materna na duração da gestação e no peso ao nascer. Mães adolescentes foram consideradas por uma pesquisa como população de maior risco para resultados desfavoráveis em relação a estes dois aspectos (Kramer, 1987); porém, não se encontrou concordância em outro estudo (Mariotoni \& Barros Filho, 1998). Ainda dentro desta discussão, existe sugestão de que a melhor maneira de se conhecer o risco reprodutivo da adolescente grávida é através da idade ginecológica. Para o Working Group on Nutrition and Pregnancy in Adolescence (Working..., 1971), a idade ginecológica baixa (menor que 2 anos) e o crescimento físico incompleto são fatores responsáveis por maior demanda nutricional durante a gravidez.

Este estudo foi, portanto, conduzido com o objetivo de investigar prováveis associações entre o uso, por adolescentes grávidas, de suplementos de ferro (diferentes concentrações), zinco e ácido fólico e o estado nutricional dos recém-nascidos.

\section{CASUISTICAE METODOS}

Os recém-nascidos estudados $(n=74$ ) eram filhos de mães adolescentes com idade entre 13 e 18 anos (média de 16 anos) de baixa renda, as quais fizeram pré-natal em Serviço Público, no Instituto de Perinatologia Social do Piauí, Maternidade Dona Evangelina Rosa (MDER), localizado no Estado do Piauí, Nordeste do Brasil, e foram suplementadas no período entre a $16^{\mathrm{a}} \mathrm{e}$ $20^{a}$ semana de gestação até o parto. Todas as jovens pesquisadas eram primíparas, com idade ginecológica de $3 \pm 1,3$ anos.

Para efeito de seleção da amostra, alguns critérios de exclusão foram adotados, tais como: a presença de complicações obstétricas ou médicas; ocorrência de aborto em um intervalo anterior à pesquisa de no mínimo seis meses; uso de qualquer hematínico e contraceptivo oral, no período de seis meses imediatamente anterior à concepção; consumo de drogas, fumo e álcool e de dietas especiais, considerados fatores geradores de divergências em estudos que investigam o papel do ferro, do zinco e do ácido fólico durante a gravidez e suas implicações no concepto. 
196 | N.N. NOGUEIRA et al.

Considerando-se ainda, possíveis reações aos medicamentos, possibilidade de eventuais esquecimentos de uso dos mesmos e falta à consulta, adotou-se como critério de permanência na pesquisa o prazo máximo de 15 dias, de forma descontínua, sem utilização dos suplementos, a contar do início da intervenção até o momento da reavaliação da adolescente.

Como as adolescentes grávidas constituem um grupo nutricionalmente de risco, procurou-se conhecer a adequação da dieta consumida pelas gestantes avaliadas através do emprego do método recordatório de 24 horas. As determinações das concentrações dos nutrientes em estudo foram realizadas através da utilização do pacote Sistema de Apoio Decisão em Nutrição (Escola Paulista..., 1990). Para o cálculo de adequação adotou-se o padrão do documento Recommended Dietary Allowances (National Research..., 1989).

Após o Comitê de Ética da Maternidade ter aprovado o protocolo da pesquisa e as adolescentes estarem cientes do estudo e concordantes com ele, estas foram aleatoriamente distribuídas em cinco grupos, para se avaliar o efeito da suplementação com ferro, zinco e ácido fólico nos seus recém-nascidos (Tabela 1).

Tabela 1. Efeito da suplementação com ferro, zinco e ácido fólico.

\begin{tabular}{cccc}
\hline Grupos & $\begin{array}{c}\text { Ferro } \\
(\mathrm{mg} / \mathrm{dia})\end{array}$ & $\begin{array}{c}\text { Ácido fólico } \\
(\mu \mathrm{g} / \mathrm{dia})\end{array}$ & $\begin{array}{c}\text { Zinco } \\
(\mathrm{mg} / \mathrm{dia})\end{array}$ \\
\hline I & 120 & 250 & 0 \\
II & 80 & 250 & 0 \\
III & 120 & 250 & 5 \\
IV & 80 & 250 & 5 \\
V & 120 & 0 & 0 \\
\hline
\end{tabular}

O composto ferro foi oferecido na forma de sulfato ferroso, administrado oralmente, em duas ou três vezes ao dia. Quanto ao ácido fólico, este foi fornecido na forma de monoglutamato, isolado ou combinado com o sulfato de zinco (ZnSO4), e tomado em dose única, associada ou não ao ferro. Por questões éticas, o grupo $V$, foi considerado controle, recebendo somente ferro, em concentrações utilizadas na rotina da instituição (120 mg/dia).

Com relação à idade gestacional das adolescentes, esta foi calculada de acordo com a data da última regra. Na incerteza desta, era feito exame de ultra-sonografia, com posterior confirmação pelo método preconizado por Capurro et al. (1978).

A tomada de peso dos recém-nascidos foi feita em balança pediátrica, com capacidade para $13 \mathrm{Kg}$ e sensibilidade de $10 \mathrm{~g}$. O comprimento foi aferido em antropômetro marca Bandeirante, aprovado pelo Instituo Nacional de Pesos e Medidas (INPM), conforme portaria 15/81, e o perímetro cefálico foi medido com fita métrica não distensível. As informações sobre as condições de nascimento da criança obtidas, através de prontuários médicos e do cartão da criança, foram organizadas em planilhas, utilizando-se o Programa Excel, versão 5.0. A análise dos dados foi feita através do Programa SPSS/PC, versão 3.0

Os dados levantados foram reunidos e estratificados de acordo com os grupos de intervenção, e controle. Foram submetidos à análise descritiva, utilizando-se tabela contendo médias e variâncias (desvio-padrão). Para efeito de comparação de valores, indivíduo a indivíduo, empregou-se o teste " $\mathrm{t}$ " Student pareado e o não pareado de Wilcoxon (Spielgel, 1988). A fim de se estimar o efeito da suplementação nos parâmetros avaliados, foram construídos intervalos com $95 \%$ de confiança. Para se verificar possíveis correlações entre as variáveis analisadas e os grupos de intervenção, também foi aplicado o teste de correlação de Pearson.

\section{RESULTADOS}

Em relação à análise da adequação da dieta consumida pelas adolescentes grávidas, a ingestão de energia e proteína encontrava-se adequada, mas as necessidades de ferro (35\%), zinco (65\%) e ácido fólico (30\%) não estavam sendo atendidas (Tabela 2). 
Quanto às características antropométricas dos recém-nascidos (Tabela 3), as médias de peso $(3.000 \pm 418 \mathrm{~g})$, comprimento $(48,07 \pm 2,15 \mathrm{~cm})$, perímetro cefálico $(33,53 \pm 1,50 \mathrm{~cm})$ e idade gestacional $(39,21 \pm 1,32$ semanas) encontradas para os filhos de mães do grupo $\vee$ (rotina local) não apresentaram diferenças significativas em comparação com os valores dos grupos de intervenção. Além disso, o baixo peso e a prematuridade não foram verificados nesta população, apesar de as mães serem adolescentes.

\section{DISCUSSÃO}

A gravidez no período da adolescência tem sido motivo de atenção e de pesquisas nos últimos anos, pois tem-se visto aumentar de forma considerável o número de gestantes com idades cada vez menores. Esta condição teve início basicamente na década de setenta, quando se verificou um aumento desmedido da atividade sexual e da gravidez entre mulheres na faixa de 13 a 19 anos de idade (Hayes, 1987).

Lippi \& Segre citado pela Organização... (1988) descreveram um estudo conduzido em São Paulo, com adolescentes entre 10 e 19 anos de idade, durante o período de 1980 a 1985, onde se observou uma elevada incidência de gestantes neste grupo (19,68\%). Destas, 25,48\% estavam com idade entre 10 e 16 anos. Recentemente, a Pesquisa Nacional de Demografia e Saúde, realizada pela Sociedade Civil Bem-Estar Familiar (BEMFAM) (Pesquisa Nacional..., 1996), demonstrou que nos últimos 10 anos a fecundidade diminuiu cerca de $30 \%$ em todas as faixas etárias, com exceção da faixa de adolescentes.

Em Teresina, local de realização deste estudo, um levantamento sobre o perfil social, psicológico, nutricional e médico de adolescentes grávidas, atendidas no Instituto de Perinatologia Social do Piauí realizado no período de julho a setembro de 1991, mostrou o proedomínio, entre as 100 adolescentes avaliadas da faixa etária de 16 a 17 anos, sendo a maioria solteira e nulípara (Carvalho et al., 1992).

Do ponto de vista biológico, vários são os riscos a que as adolescentes grávidas estão expostas. Segundo Camarano (1998), este grupo apresenta uma incidência maior de complicações obstétricas, incluindo: hipertensão específica da gravidez, prematuridade, desproporção céfalo-pélvica, trabalho de parto prolongado e aumento

Tabela 2. Composição e adequação da dieta consumida pelas adolescentes grávidas, de acordo com o padrão de referência da National Academy of Sciences (RDA - 1989).

\begin{tabular}{lccc}
\hline Componentes da dieta & Ingestão média diária & Ingestão recomendada & Percentual de adequação \\
\hline Energia & $1900 \pm 694,0$ & 2500 & 79,6 \\
Proteína $(\mathrm{g})$ & $81,8 \pm 29,7$ & 60 & 122,2 \\
Ferro $(\mathrm{mg})$ & $10,5 \pm 4,6$ & 30 & 35,0 \\
Zinco $(\mathrm{mg})$ & $9,8 \pm 5,0$ & 15 & 65,0 \\
Folato $(\mu \mathrm{g})$ & $119,7 \pm 82,0$ & 400 & 30,0 \\
\hline
\end{tabular}

Tabela 3. Características antropométricas de recém-nascidos de mães adolescentes, de acordo com os grupos de intervenção e controle. Instituto de Perinatologia Social do Piauí, MDER

\begin{tabular}{cccccc}
\hline Grupos & Amostra & Peso (grama) & Comprimento (cm) & Perimetro Cefálico (cm) & $\begin{array}{c}\text { Idade Gestacional } \\
\text { (semanas) }\end{array}$ \\
\hline I & 15 & $3,159 \pm 436$ & $48,60 \pm 2,47^{*}$ & $33,86 \pm 1,35$ & $39,27 \pm 1,23$ \\
II & 13 & $3,286 \pm 394$ & $48,90 \pm 2,43$ & $34,10 \pm 1,50$ & $40,00 \pm 1,15$ \\
III & 14 & $3,193 \pm 278$ & $48,60 \pm 1,44$ & $34,00 \pm 0,75$ & $39,00 \pm 0,86$ \\
IV & 17 & $3,323 \pm 455$ & $48,81 \pm 1,70$ & $34,46 \pm 1,31$ & $39,84 \pm 1,22$ \\
V & 13 & $3,000 \pm 418$ & $48,07 \pm 2,15$ & $33,53 \pm 1,50$ & $39,21 \pm 1,32$ \\
\hline
\end{tabular}

(*) Médiae desvio-padrão. 
198 | N.N. NOGUEIRA et al.

da perda perinatal. Tais complicações continuam sendo verificadas por alguns autores (Carvalho et al., 1992; Scholl et al. 1992; Scholl \& Hediger, 1993); porém, atualmente, outros discordam dela (Mariotoni \& Barros Filho, 1998).

Os maiores riscos de baixo peso ao nascer são encontrados entre bebês de mães adolescentes. Da mesma forma, também eleva-se neste grupo a incidência de partos prematuros. Por outro lado, quanto às prováveis associações entre distúrbios nutricionais em jovens grávidas e estas complicações, os dados disponíveis são bastante conflitantes (Tamura et al., 1992; Stephenson, 1995).

No tocante às deficiências nutricionais específicas, pesquisas para avaliar fatores de risco de retardo no crescimento fetal mostraram que a suplementação com o ácido fólico, por exemplo, reduziu a prevalência deste distúrbio e de infecção materna e teve efeito favorável sobre o peso ao nascer e Apgar de recém-nascidos (Tamura et al., 1992). De acordo com estudos prévios, utilizando folato adicionado ao ferro suplementar, esta associação não só aumentou o peso ao nascer como também diminuiu a incidência de prematuridade em pacientes com deficiência de folato (Baumslag et al., 1970).

Quanto ao zinco, em contraste com os inúmeros trabalhos sobre os efeitos maléficos de sua deficiência materna em animais experimentais, existem poucas informações relacionadas à espécie humana. No entanto, existem dados sugestivos de riscos semelhantes (Hambidge et al., 1983) Apesar disso, a posição de outros autores sobre o tema é de que o uso terapêutico do zinco em mulheres grávidas para melhorar o peso ao nascer ainda não está bem estabelecido (Islam et al., 1994; Caulfield et al., 1999).

Ao contrário de relatos descritos neste artigo a respeito de complicações, sobretudo a elevada incidência de baixo peso ao nascer e de prematuridade, os recém-nascidos avaliados nesta pesquisa, cujas mães foram suplementadas com ferro, em diferentes concentrações, zinco e ácido fólico, apresentaram um bom perfil nutricional, com indicadores antropométricos satisfatórios. Além disso, não foram observadas diferenças significativas no estado nutricional dos recém-nascidos dos grupos de intervenção em comparação com os do grupo $V$ (rotina da instituição), no qual as adolescentes receberam apenas ferro, mineral cuja deficiência está intimamente relacionada a distúrbios maternos e perinatais.

Estes resultados, podem ser atribuídos, em parte, a determinadas características das adolescentes estudadas, tais como a idade (68\% delas tinham mais de 16 anos) e a idade ginecológica (superior a 2 anos) fatores considera-dos de grande risco para as variáveis analisadas nesta pesquisa. Destaca-se também, entre os possíveis determinantes dos dados obtidos, o relevante papel de uma boa assistência pré-natal na redução de complicações na mãe e no concepto.

\section{AGRA DECIMENTOS}

Às adolescentes. Aos médicos: Vicente de Paula Canapum, Márcia S.J. Martins e Carolina dos S. Oliveira. Às alunas de iniciação científica Dilina do N. Marreiro, Márcia Andréia Moura Fé, Adriana e Patricia e a Fabiana Poltroniere.

\section{REFERENCIAS BIBLIOGRÁFICAS}

APGAR, J. Zinc and reproduction: an update. Journal Nutrition Biochemistry, Stoneham, v.3, n.3, p.266-278, 1992.

BAILEY, B., CERDA, J.J. Iron and folate nutriture during life cycle. World Review of Nutrition and Dietetics, London, v.56, p.56-92, 1988.

BAUMSLAG, N., EDELSTEIN, T., METZ, J. Reduction of incidence of prematurity by folic acid supplementation in pregnancy. British Medical Journal, London, v.1, n.687, p.16-17, 1970.

BLOT, I., DIALLO, D., TCHERNIA, G. Iron deficiency in pregnancy: effects on the newborn. Current 
Opinion in Hematology, Philadelphia, v.6, n.2, p.65-70, 1999.

CAMARANO, A.A. Fecundidade e anticoncepção da população de 15 a 19 anos. In: SEMINÁRIO GRAVIDEZ NA ADOLESCÊNCIA, 1998, Rio de Janeiro. Anais... Rio de Janeiro : USAID, 1998. p.35-46.

CAPURRO, H., KONICHEZKY, S., FONSECA, D., CALDEYRO-BARCIA, R. A simplified method for diagnosis of gestational age in the newborn infant. Journal of Pediatrics, St. Louis, v.93, n.1, p.120-122, 1978.

CARVALHO, E.M.M.O., COSTA, M.S.V., SAMPAIO, C.R., MOURA FÉ, M.B.L., SANTOS, M.R., MARTINS, M.S.J., PARENTE, J.V. Indicadores de risco gestational no Piauí. Revista do Hospital Getúlio Vargas, Teresina, v.1, n.2, p.84-87, 1992.

CAULFIELD, L.E., ZAVALETA, N., FIGUEROA, A., LEON, Z. Maternal zinc supplementation does not affect size at birth or pregnancy duration in Peru. Journal Nutrition, Bethesda, v.129, n.8, p.1563-1568, 1999.

DALY, L.E., KIRKE, P.N., MOLLOY, A., WEIR, D.G., SCOTT, J.M. Folate levels and neural tube defects: Implications for prevention. JAMA, Chicago, v.274, n.21, p.1698-1718, 1995.

ESCOLA PAULISTA DE MEDICINA. Centro de Informação em Saúde. Sistema de avaliação nutricional. São Paulo, 1990. [Software].

HAMBIDGE, K.M., KREBS, N.F., JACOBS, M.A., FAVIER, A.G., IKLE, D.N. Zinc nutricional status during pregnancy: a longitudinal study. American Journal of Clinical Nutrition, Bethesda, v.37, n.3, p.429-442, 1983.

HAYES, C.D. (Ed.). Risking of the future: adolescent sexuality, pregnancy, and childbearing. Washington DC : National Academy Press, 1987. v.1.

HEDIGER, M.L., SCHOLL, T.O., KHOO, C.S., FISCGER, R.F. New research findings in adolescent health. Journal Adolescent Health, New York, v.13, p.46, 1992.

ISLAM, M.A., HEMALATA, P., BHASKARAM, P., KUMAR, P.A. Leukocyte and plasm zinc in maternal and cord blood: their relationship to period of gestation and birth weight. Nutrition Research, New York, v.14, n.3, p.353-360, 1994.

KRAMER, M.S. Determinants of low birth weight: methodological assessment and meta-analysis. Bulletin, of the World Health Organization, Geneva, v.65, n.5, p.663-637, 1987.

MARIOTONI, G.G.B., BARROS FILHO, A.A. A gravidez na adolescência é fator de risco para o baixo peso ao nascer? Jornal de Pediatria, Rio de Janeiro, v.74, n.2, p.107-113, 1998.

NATIONAL RESEARCH COUNCIL (USA). Recommended dietary allowances. 10.ed. Washington DC: National Academy of Sciences, 1989. 284p.

ORGANIZAÇÃO PAN-AMERICANA DA SAÚDE. Coletânea sobre saúde reprodutiva do adolescente brasileiro. Brasília, 1988. 255p.

ORGANIZACIÓN MUNDIAL DE LA SALUD. Necesidades de vitamina A, hierro, folate y vitamina B12. Geneva, 1987. p.61-73.

PESQUISA sobre saúde e demografia. Relatório BEMFAN. Rio de Janeiro, 1996. 47p.

PREZIOSI, P., PRUAL, A., GALAN, P., DAOUDA, H., BOUREIMA, H., HERCBERG, S. Effect of iron supplementation on the iron status of pregnant women: consequences for newborns. American Journal of Clinical Nutrition, Bethesda, v.66, n.5, p.1178-1182, 1997.

ROTHMAN, D. Folic acid in pregnancy. American Journal of Obstetrics Ginecology, St. Louis, v.108, n.1, p.149-175, 1970

SANDSTEAD, H.H. Zinc in human nutrition. In: Disorders of mineral metabolism: trace minerals. New York : Academic Press, 1981. v.1, p.94-157.

SCHOLL, T.O., HEDIGE, R.M.L., FISCHER, R.L, SHEARER, J.W. Anemia vs iron deficiency: increased risk of preterm delivery in a prospective study. American Journal of Clinical Nutrition, Bethesda, v.55, n.5, p.985-988, 1992.

SCHOLL, T.O., HEDIGER, M.L. A review of the epidemiology of nutrition and adolescent pregnancy: maternal growth during pregnancy and its effect on the fetus. Journal of the American 
200 | N.N. NOGUEIRA et al.

College of Nutrition, New York, v.12, n.2, p.101-107, 1993.

SEVER, L.E., EMANUEI, I. Is there a connection between maternal zinc deficiency and congenital malformations of the central nervous system in man? Teratology, New York, v.7, n.1, p.117, 1973.

SPIELGEL, S. Estatística não paramétrica. São Paulo : McGraw-Hill do Brasil, 1988. 354p.

STEPHENSON, L.S. Possible new developments in community control of iron-deficiency anemia. Nutrition Reviews, New York, v.53, n.2, p.23-30, 1995.

TAMURA, T., GOLDENBERG, R., FREEBERG, L.E., CLIVER, S.P, CUTTER, G.R., HOFFMAN, H.J. Maternal serum folate and zinc concentration and their relationships to pregnancy outcome. American Journal of Clinical Nutrition, Bethesda, v.56, n.2, p.365-370, 1992.

WORKING group on nutrition and pregnancy in adolescence. Relation of nutrition to pregnancy in adolescence. Clinical Obstetrics and Gynecology, Philadelphia, v.14, n.2, p.367-390, 1971.

YIP R. The challenge of controlling iron deficiency: sweet news from Guatemala. American Journal of Clinical Nutrition, Bethesda, v.61, n.5, p.1164-1165, 1995.

Recebido para publicação em 14 de novembro de 2000 e aceito em 3 de setembro de 2001. 